\title{
Unusual morphology of equimolar Ar-Kr alloys
}

\author{
V.V. Danchuk, A.A. Solodovnik, N.S. Mysko, and M.A. Strzhemechny \\ B. Verkin Institute for Low Temperature Physics and Engineering of the National Academy of Sciences of Ukraine \\ 47 Lenin Ave., Kharkov 61103, Ukraine \\ E-mail: danchuk@ilt.kharkov.ua
}

Received February 26, 2015, published online April 23, 2015

\begin{abstract}
The transmission high electron energy diffraction (THEED) technique was employed for studying the structure of the equimolar $\mathrm{Ar}-\mathrm{Kr}$ alloy, in which the thermodynamics predicts the maximum feasibility of phase separation. Deposition of preliminarily cooled gas mixtures was performed onto substrates cooled to 6 or $20 \mathrm{~K}$. All diffraction patterns contained several sets of reflections against an appreciable background. Analysis of the data obtained during a warm-up from 6 to $33 \mathrm{~K}$ (at which the major part of argon was removed due to sublimation) as well as of the diffraction pattern from the "sandwich" (two successively deposited film of pure Ar and $\mathrm{Kr}$ ) provided reliable arguments for the following conclusions. Actually, we have documented for the first time a phase separation of an $\mathrm{Ar}-\mathrm{Kr}$ mixture, manifestations of which turned out to be oddly asymmetric as far as the behavior of the components involved is concerned. Upon deposition both onto 6 or $20 \mathrm{~K}$ the emerging sample contained two crystal phases of virtually pure argon with a small admixture of krypton. One of the Ar phases (fcc) did not cause a surprise, whereas the other was hcp with the $a / c$ ratio close to the ideal value. The krypton component separated as a fine-grained glass-like state, possibly, with a low admixture of argon.
\end{abstract}

PACS: $61.05 . J-\quad$ Electron diffraction and scattering;

78.55.Kz Solid organic materials;

61.66.Dk Alloys.

Keywords: electron diffraction, $\mathrm{Ar}-\mathrm{Kr}$ alloys, morphology.

\section{Introduction}

Rare gas binary alloys are suitable objects for investigation into basics of solid state [1]. These systems can be consistently described using the Lennard-Jones pairwise potential, which enables a proper testing of relevant theoretical approach, in particular, data concerning liquid and solid rare gas mixtures formed a basic of the theory dealing with formation and stability of binary mixtures. According to Prigogine [2], for any composition of a solid binary solution there is a critical temperature, below which phase separation is possible. This critical temperature is highest for equimolar solutions. Structure studies of $\mathrm{Ar}-\mathrm{Kr}$ alloys at liquid helium temperatures [3,4] gave evidence of complete mutual solubility which contradicts basic thermodynamics laws. Arguments for the statement of mutual solubility in the solid over the entire composition range were based on the observation that the composition dependence of the lattice parameter agrees well with Vegard's and Prigogine's rules. First evidence for incomplete solubility in $\mathrm{Ar}-\mathrm{Kr}$ alloys was documented in neutron scattering studies [5]. Analysis brought the authors to the conclusion that the
$\mathrm{Kr}$ matrix contains Ar clusters, which means that the inferable of the complete solubility of both components has to be but to doubt. The boson peak discovered in calorimetric studies on the $\mathrm{Kr}-25 \%$ Ar alloy [6] was another evidence against the homogeneity of $\mathrm{Ar}-\mathrm{Kr}$ alloys within the entire range of mutual concentrations. A similar conclusion about the complete solubility was made for the $\mathrm{N}_{2}-\mathrm{Ar}$ alloys (a close analog of the $\mathrm{Ar}-\mathrm{Kr}$ system) [7,8]. However in later investigations $[9,10]$ the separation boundaries have been determined for this binary system. The principal discrepancy between the conclusions of the two groups concerning the $\mathrm{N}_{2}-\mathrm{Ar}$ alloys can be explained by the differences in sample preparation conditions and procedures.

It should be remarked that it is the possibility to vary in a controlled way the morphology of emerging samples by small changes in preparation conditions that constitutes the basis of the present day methods employed in synthesizing nanostructured materials [11]. One of the physical methods used for preparation of nanostructures is by direct deposition. It is this method that we employed as the preparation technique for growing $\mathrm{Kr}-\mathrm{Ar}$ cryoalloys over the entire 
range of mutual concentrations and within a broad range of varying deposition parameters. In this paper we present results of transmission high energy electron diffraction (THEED) studies of $\mathrm{Kr}-\mathrm{Ar}$ samples grown from equimolar gas mixtures preliminarily cooled on their way along the feeding tube kept at temperatures slightly below the liquidnitrogen point (sub-LN temperatures) and show that their morphology differs drastically from, that of a regular alloy.

\section{Experimental}

Argon-krypton alloys were studied using the THEED method on a EG-100A electron diffraction setup equipped with a liquid-helium cryostat. Samples were prepared directly inside the working column by deposition gas mixtures of known composition onto a cooled substrate film [12]. The gases were mixed in a special bottle at room temperature, the typical mutual diffusion time according to the Einstein-Smoluchowski relations amounted to $21 \mathrm{~s}$. Therefore, the typical waiting time of up to 5 minutes at room temperature ensured a uniform distribution of the gases. The net pressure in the bottle did not exceed 12 Torr. The actual composition was calculated from the initial partial pressures of the components. Preparation of samples was carried out using very small amounts (less than $0.5 \mathrm{~cm}^{3}$ ) of the gas mixture; leak-in was performed in the laminar regime. The design of the cryoshields allowed variations of the inlet tube temperature from room to subLN, which helped us to vary the morphology of emerging deposits within a broad range.

Gas mixtures were deposited onto a polycrystalline aluminium substrate. The overall level of impurities in the source argon and krypton gases did not exceed $0.01 \%$. When samples grew to a large-grain and homogenous films, the lattice parameter error was below $0.1 \%$. The samples were grown by depositing equimolar $\mathrm{Ar}-\mathrm{Kr}$ gas mixtures onto a film substrate cooled down to 6 or $20 \mathrm{~K}$, the tip of the filling tube being kept at sub-LN temperature.

The THEED patterns were recorded on photo plates either after the sample was deposited at a proper temperature or during a subsequent heating of the samples up to the relevant sublimation temperatures. The duly developed patterns were digitized using a CanoScan 9950F scanner with resolution $1200 \mathrm{dpi}$, the maximum optical density of the scanner being 3.8 D. Digitized THEED patterns were processed using Figi ImageJ package. The average size of coherent scattering regions was evaluated using the true diffraction line broadening [13] with account of stacking faults $[14,15]$ and making use of the Selyakov-Scherrer relationship [16] for globular crystallites, which enabled us to deal with mean grain sizes. The stacking fault density was evaluated from the shifts of diffraction peaks respective their "reference" positions calculated from the position of line 311 [17].

\section{Results and discussion}

Deposition of sub-LN equimolar $\mathrm{Ar}-\mathrm{Kr}$ gas mixtures onto a $6 \mathrm{~K}$ substrate resulted in polycrystalline weakly textured layers as shown in Fig. 1. We failed to determine the structure and morphology of this sample within a monophase assumption. Besides the reflections with inter-plane distances $3.062,2.656,1.882$, and $1.602 \AA$, which correspond to indices, respectively, (111), (200), (220), and (311) of a fcc Ar structure with a lattice parameter of $5.323 \AA$, the pattern contained reflections at small angles with inter-plane distances $d_{1}=3.249 \AA\left(S_{1}=1.934 \AA^{-1}\right)$ and $d_{2}=2.854 \AA\left(S_{2}=2.201 \AA^{-1}\right)$, which needed a special analysis. It should be noted here that reflex $d_{2}$, because of its low intensity and large spread, was identified poorly so that its very existence caused doubt. In Fig. 2 we present a densitogram of the pattern plotted in Fig. 1. Subtraction of the noncoherent background revealed that, apart from reflections fcc Ar and $d_{n}$, the pattern includes a rather intensive halo at small diffraction angles around $S=2.16 \AA^{-1}$. Let us discuss available possibilities to explain the $d_{n}$ system and the halo.

Assumption 1: the $d_{n}$ reflection belong to a Kr-rich fcc phase (reflections (111) and (200), respectively). If so, $d_{1}$ yields the lattice parameter $5.627 \AA$, which could belong to a $\mathrm{Kr}-10 \% \mathrm{Ar}$ alloy. Reflection $d_{2}$ corresponds to $a=$ $=5.719 \AA$, which by $0.05 \AA$ exceeds the value for pure krypton fcc $\left(a_{\mathrm{Kr}}=5.67 \AA\right)$. Subsequent annealing of this sample up to $20 \mathrm{~K}$ entailed brightening of $d_{n}$ reflections. The weak texture of the sample helped to "backlight" the small-angle reflections, which allowed undoubted detection and localization of the $d_{2}$ (Fig. 3).

The evident inconsistencies (positions and intensities) of the $d_{n}$ reflections as representing the above-mentioned Kr-rich phase, as well as the absence of the reflection with $d_{3}=1.709 \AA\left(S_{3}=3.676 \AA^{-1}\right)$, which is characteristic of the $\mathrm{Kr}$ (311) reflection, constitute a solid argument for the claim that the $d_{n}$ system cannot belong to a krypton-rich fcc phase. A clear example how two fcc structures overlap is the pattern in Fig. 4 from the $\mathrm{Ar}-\mathrm{Kr}$ "sandwich". This "sandwich" sample was produced in the following way.

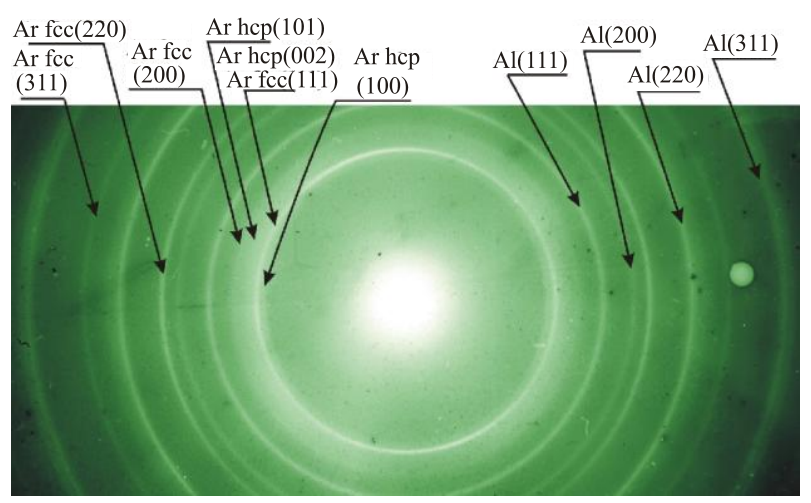

Fig. 1. (Color online) Diffraction pattern of equimolar $\mathrm{Ar}-\mathrm{Kr}$ sample. Deposition and registration temperatures are $6 \mathrm{~K}$. 


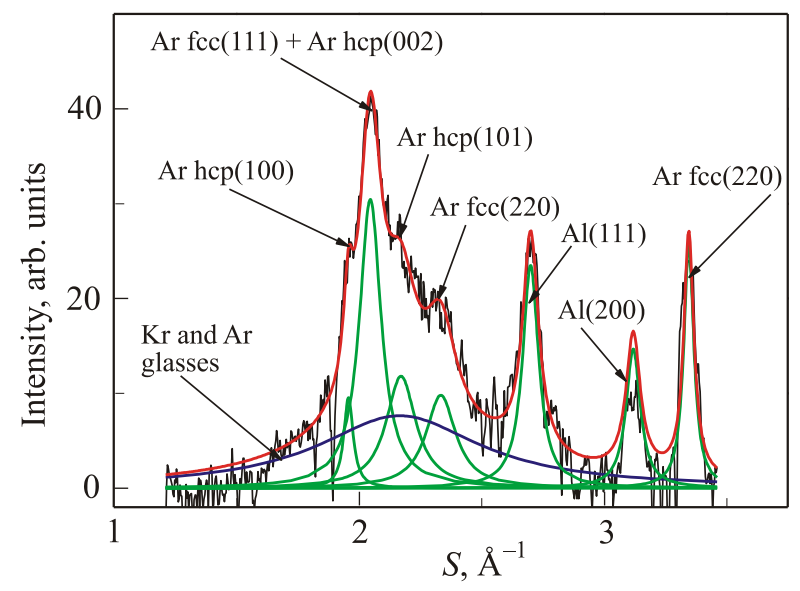

Fig. 2. (Color online) The diffracted intensity versus diffraction parameter $S=4 \pi \sin \theta / \lambda$ ( $\theta$ is the Bragg angle and $\lambda$ is the electron wave length) for the pattern in Fig. 1.

First, a pure krypton film was deposited at $20 \mathrm{~K}$, then above it a pure argon film. In Fig. 4 the fcc peaks of both Ar and $\mathrm{Kr}$ fcc phases nicely separate; the (111)/(200) intensity ratios agree, within a small error, with the calculated values for fcc phases of $\mathrm{Ar}$ and $\mathrm{Kr}$. Notice that the fcc $\mathrm{Kr}$ reflections are brighter than those of the Ar phase, which correlates with the atomic $\mathrm{Ar} / \mathrm{Kr}$ scattering factor ratio.

Assumption 2: $d_{n}$ reflections are due to secondary diffraction from growth twin boundaries. In principle, the $d_{n}$ reflections in Figs. 2 and 3 could be explained as a results of secondary diffraction from growth twins [18]. Making use of the shifts of the (111) and (200) peaks of the argonrich phase from their reference positions relative to the (311) reflection we evaluated the deformational and growth stacking fault densities $[14,15]$. The rather small fault density parameters $(\alpha \leq 0.015$ and $\beta \leq 0.06)$ are insufficient to ensure formation of an extra reflection. Another independent argument against the assumption under dis-

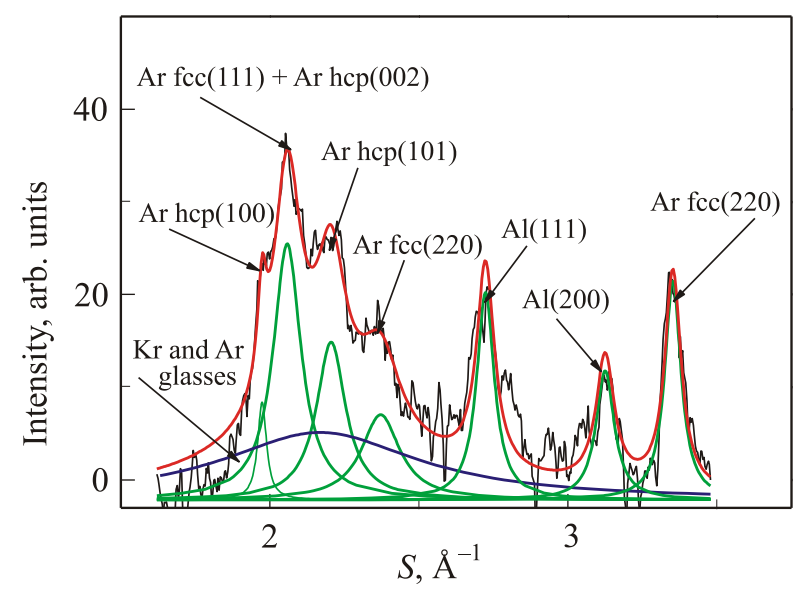

Fig. 3. (Color online) Densitogram of annealed equimolar $\mathrm{Ar}-\mathrm{Kr}$ sample deposited on $6 \mathrm{~K}$ and registered at $20 \mathrm{~K}$.

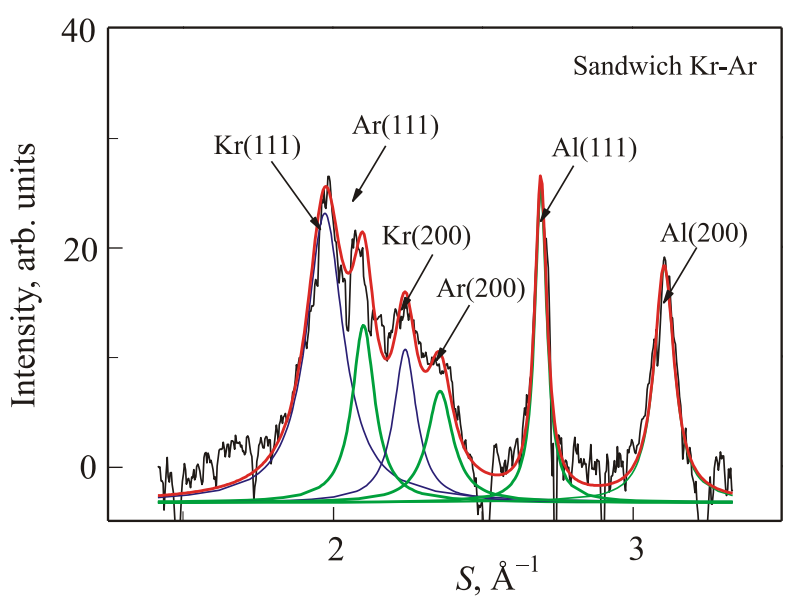

Fig. 4. (Color online) Densitogram of the $\mathrm{Ar}-\mathrm{Kr}$ sandwich.

cussion is the presence of the $d_{2}$ peak, which does not appear due to boundary diffraction [18].

Assumption 3: $d_{n}$ reflections are formed by the hcp phase of almost pure Ar. Upon indexing $d_{1}$ and $d_{2}$ as (100) and (101) reflections of the hcp phase and taking into account the inevitable superposition of reflections (111) and (002) from, respectively, the fcc and hcp phases we have calculated the hcp lattice parameters to be: $a=3.752 \AA$, $c=6.104 \AA, c / a=1.630$. These values are somewhat larger than those for pure argon, which is owing presumably to the presence of some atoms of krypton in the Ar hcp phase. The (102) reflection of the hcp argon phase is superimposed on the Al reference reflection (111) (which can be in general absent). Van de Waal et al. [19] presented a model diffraction pattern from a hep polycluster of eight disoriented domains. Account of the intensity scattered from inter-grain boundaries entailed suppression of certain hcp lines and, in particular, disappearance of line (102). The calculated spectra [19] were compared with experimental diffraction data for free argon clusters to show that in some cases the (102) reflection is not necessary for the identification of hcp phases. Formation of hcp phase together with the prevailing fcc phase as a consequence of annealing of cold-deposited pure $\mathrm{Ar}$ and $\mathrm{Ne}$ films at temperatures close to sublimation had been documented by Kovalenko and Bagrov [14].

In Fig. 5 we show a diffraction pattern from a sample of grown from sub-LN argon on a substrate at $20 \mathrm{~K}$. Only the fcc reflections are present, the lattice parameter being $a=$ $=(5.322 \pm 0.005) \AA$. The stacking fault densities $\alpha=0.018$ and $\beta=0.059$ are comparable with those in the equimolar $\mathrm{Ar}-\mathrm{Kr}$ samples. It is more interesting that the average grain size in pure Ar about $70 \AA$, as evaluated with account for stacking faults, is by a quarter less than the grain size of the fcc phase in the equimolar samples, about $100 \AA$. This is presumably a consequence of a higher mobility of argon atoms in the crystallizing layers due to the elevated latent heat of the krypton in the equimolar samples. In addition, the very crystal growth mechanisms during deposition of 


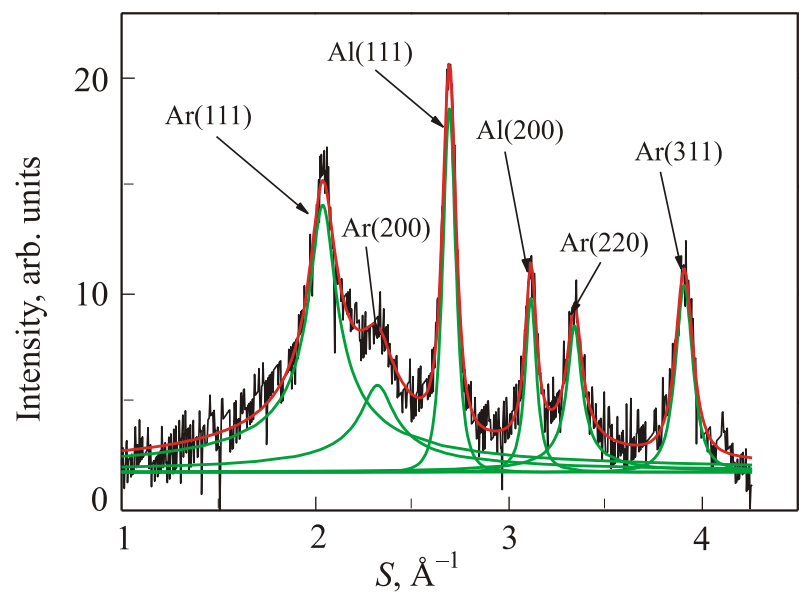

Fig. 5. (Color online) Densitogram of pure Ar deposited and registered at $20 \mathrm{~K}$.

the equimolar Ar- $\mathrm{Kr}$ mixtures and the pure argon differ. For pure Ar the nucleus formation mechanism is mostly homogeneous. But during deposition of $\mathrm{Ar}-\mathrm{Kr}$ mixtures, the krypton atoms and small clusters can serve as condensation centers for Ar atoms. Therefore, nucleation mechanism will be basically heterogeneous and, as a consequence, the coherent scattering entities will have larger sizes.

After the equimolar samples were heated up to $20 \mathrm{~K}$, the grain size of the fcc phase (about $90 \AA$ ) stayed actually unchanged while the grain size of the hcp phase grew up almost by one third, from 75 to $110 \AA$, making the relevant reflections more distinct (cf. Fig. 3).

Deposition of a sub-LN Ar-Kr equimolar gas mix onto a "warm" substrate at $20 \mathrm{~K}$ resulted in samples with patterns like shown in Fig. 6. The fcc reflections grew brighter and sharper compared to cold-deposited samples, the mean grain size (of about $105 \AA$ ) are eventually the same as in colddeposited equimolar samples. It is noteworthy that stacking faults in "warm"-deposited samples are denser: we evaluated the fault parameters to be $\alpha=0.064 ; \beta=0.087$ (we remind that in cold-deposited samples these quantities were $\alpha \leq 0.015 ; \beta \leq 0.06$ ). This seeming paradox can be explained as follows: it is known that hcp nuclei in a fcc matrix are, in particular, stacking faults. Therefore, since the hcp fraction in cold-crystallized samples is higher than in warmdeposited samples, a larger amount at stacking faults are "eaten up" by the hcp regions present.

Analysis of the diffraction pattern in Fig. 6 unambiguously indicates presence of reflections which are characteristic of a hcp structure. It is well known that the diffraction intensity is proportional to the scattering volume, which allows estimation of the hcp/fcc ratios in our samples. The ratio $R=I_{101} / I_{200}$ in warm-deposited samples $R=1.33$ turns out to be below the corresponding estimate for colddeposited sample where $R=1.81$, that is, the amount of hcp in warm-deposited samples is smaller than in colddeposited ones. The overage hcp grain size in warm-

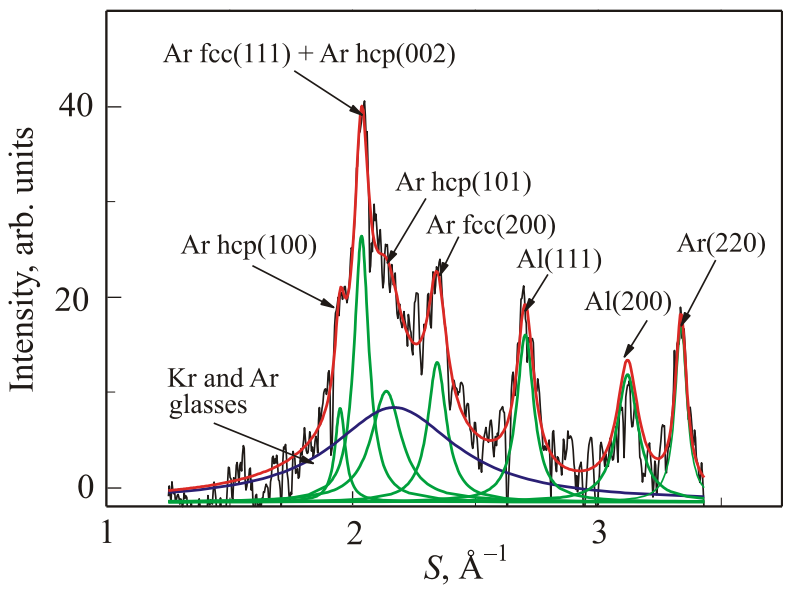

Fig. 6. (Color online) Diffraction pattern from an equimolar $\mathrm{Ar}-\mathrm{Kr}$ sample grown and registered on a "warm" substrate at $20 \mathrm{~K}$.

deposited sample was estimated to be $116 \AA$ or, in other word, it is the same within the error with the hcp grain size in cold-deposited sample after it was warmed up to $20 \mathrm{~K}$. The fact that higher stacking faults in the fcc phase in samples with lower hcp contents correlates with the similar conclusions for large free argon clusters [20]. The abovestated fact, viz., the data concerning deposition of sub-LN argon as well as sub-LN equimolar $\mathrm{Ar}-\mathrm{Kr}$ mixtures onto the substrate at $20 \mathrm{~K}$ give us grounds to the assert that it is the presence of krypton in the mix that qualitatively influences the morphology of the emerging hcp grains during deposition of sub-LN equimolar $\mathrm{Ar}-\mathrm{Kr}$ mixtures. The substrate temperature plays a subsidiary role.

The only question left unresolved is where krypton, which constitutes half of the gas mixture deposited, "disappears". According to rough estimates, both (fcc and hcp) crystal phases contain krypton in amounts less than 10 mol\%. To answer this question, both cold - deposited and warm-deposited samples were heated further. Within the argon sublimation range, from 30 to $32 \mathrm{~K}$, the peaks in diffraction patterns disappeared, resulting in a pattern like that shown in Fig. 7. The densitogram contains diffraction peaks from the aluminum reference (substrate) with a halo around $S=1.97 \AA^{-1}$. Since at $33 \mathrm{~K}$ argon had been removed, its characteristic crystal lines are absent. Further warmup to $40 \mathrm{~K}$ resulted in a narrowing of the halo feature as well as in appearance (against that background) of a weak fcc reflections (111) and (311) of pure krypton. Subsequent warmup to $42 \mathrm{~K}$ entailed a "sweeping" of the substrate, leaving in the diffraction pattern only reflections, which belong to fcc aluminum. The above heatingprovoked transformation was identical both for colddeposited and warm-deposited samples. Thus, the abovementioned halo is directly related with the krypton contained in the pre-cooled gas mixture. A quite similar situation with the "disappearance" of one of the components from coherent part of the diffraction pattern was observed in experiments [21] with $\mathrm{Kr}-\mathrm{CO}_{2}$ alloys. The correspond- 


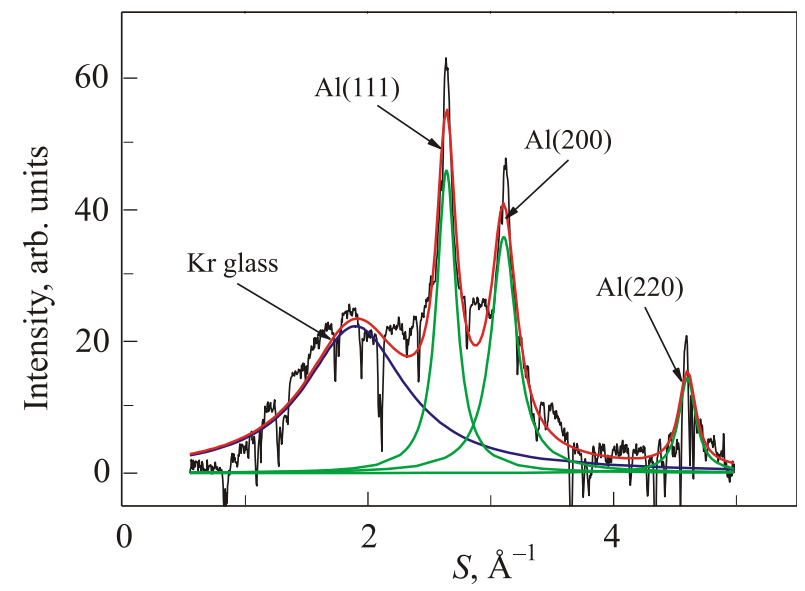

Fig. 7. (Color online) Diffraction pattern from an equimolar $\mathrm{Kr}-\mathrm{Ar}$ sample warmed from 20 to $33 \mathrm{~K}$.

ing analysis led to the conclusions that the "lost" component (carbon dioxide) was present in the form of ultradispersed inclusions. Such morphology is typical of dispersion hardened alloys; nanosize inclusions become a factor that determines unique physical properties of these novel materials [22].

As known, the very procedure of the best choice for the functions that describe the profiles of the patterns can provide information concerning the morphology of the entities under study [23]. According to Guinier [16], the appropriate function to approximate diffraction peaks in an ideal crystal is the Gauss function. It turned out that the shapes of the peaks discussed in this article (Figs. 4 through to 7) the best fitting function within the $1.5 \AA^{-1} \leq S \leq 2.5 \AA^{-1}$ range was the Lorentzian whereas the fcc peak (220) and all reference peaks are described best by the Gaussian. Analysis of the shapes of diffraction peaks from free argon clusters [24] led to a conclusion that the Lorentzian shape associates with higher densities of micro distortions caused

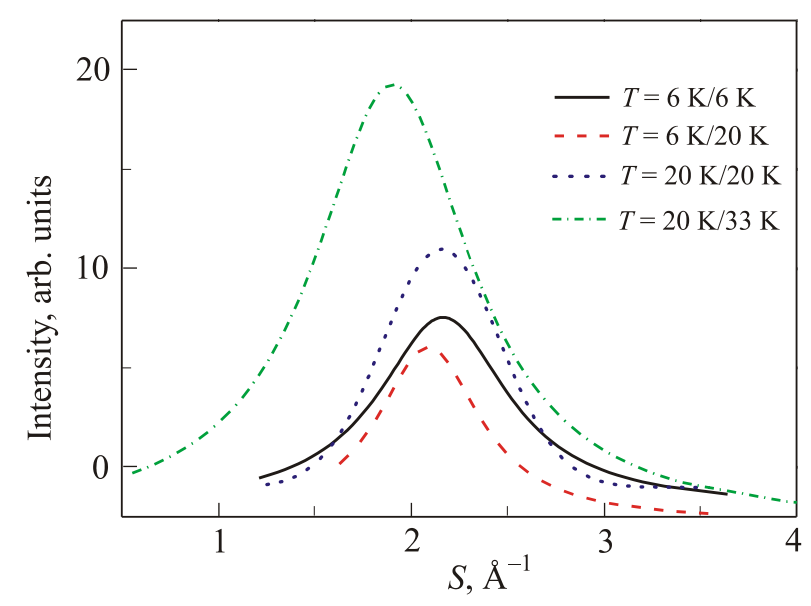

Fig. 8. (Color online) Variation of the krypton halo with temperature. by the icosahedron ordering in small argon cluster, the presence of five-fold symmetry, etc.

Unlike the pattern from pure argon (cf. Fig. 5), the smaller-angle part of which, namely, reflections (111) and (200), fit well the Lorentzian shape, the fitting of the pattern from equimolar samples necessitates modelling not only the four coherent reflections but also the halo. In Fig. 8 we show how this halo transforms with increasing temperature (the intensities are not related to one another). The preparation procedure virtually did not affect the position and width of the halo (the solid dotted curves in Fig. 8). The average grain sizes were close to $10 \AA$. After argon was removed by warming to $33 \mathrm{~K}$, the center of halo (green tallest curve) moved from $S=2.16 \AA^{-1}$ to $S=1.97 \AA^{-1}$, i.e., close to the position of the fcc (111) peak from pure krypton. It is noteworthy that after argon purging the halo grew evidently broader. Therefore, in addition to krypton atoms, argon atoms also participate in the formation of the halo.

\section{Conclusions}

The samples of argon-krypton alloys studied by THEED were prepared by direct deposition of equimolar gas mixtures, preliminarily cooled (as explained above), onto substrates at 6 and $20 \mathrm{~K}$. The entire set of reflections in all diffraction patterns could not be interpreted as belonging to any unique space group. Analysis showed that the diffraction pattern contains reflections from two crystalline phases (a fcc $\mathrm{Ar}-5 \% \mathrm{Kr}$ solution and a hcp phase of almost pure argon) and a broad halo, which corresponded to a glass-like phase of almost pure krypton. Summing up, we managed for the first time to prepare a situation to ensure a phase separation of an equimolar argon-krypton alloy. The unexpected result was the fact that the separated phases possessed crucially different morphologic forms: the krypton-rich phase was basically in a nanocrystalline (amorphous) state, whereas the predominantly argoncontaining phase included two crystalline states, fcc and hcp.

The authors express their gratitude to A.V. Dolbin for the high-purity gases used in these studies. We also thank S.I. Kovalenko for critical reading of the manuscript.

1. Rare Gas Solids, M.L. Klein and J.A. Venables (eds.), Academic Press, London, (1976) vol. 1; (1977) vol. 2.

2. I. Prigogine, The Molecular Theory of Solutions, NorthHolland, Amsterdam (1957).

3. A.E. Curzon and A.J. Mascall, J. Phys. C 2, 220 (1969).

4. S.I. Kovalenko, E.I. Indan, and A.A. Khudoteplaya, Phys. Status Solidi A 13, 235 (1972).

5. H.M. Gilder, Phys. Rev. Lett. 61, 2233 (1988).

6. M.I. Bagatskii, S.B. Feodosiev, I.A. Gospodarev, O.V. Kotlyar, E.V. Manzhelii, A.V. Nedzvetskiy, and E.S. Syrkin, Fiz. Nizk. Temp. 33, 741 (2007) [Low Temp. Phys. 33, 564 (2007)].

7. C.S. Barrett and L. Meyer, J. Chem. Phys. 42, 107 (1965). 
8. A.E. Curzon and M.S. Eastell, J. Phys. 4, 689 (1971).

9. S.I. Kovalenko, I.N. Krupskii, and A.A. Solodovnik, Fiz. Nizk. Temp. 2, 781 (1976) [Sov. J. Low Temp. Phys 2, 285 (1976)].

10. L.D. Yantsevich, A.I. Prokhvatilov, I.N. Krupskii, and A.S. Barylnik, Fiz. Nizk. Temp. 12, 300 (1986) [Sov. J. Low Temp. Phys. 12, 170 (1986)].

11. C.P. Poole, Jr. and F.J. Owens, Introduction to Nanotechnology, John Wiley \& Sons, Hoboken, New Jersey (2003).

12. A.A. Solodovnik, V.V. Danchuk, and N.S. Mysko, Fiz. Nizk. Temp. 39, 586 (2013) [Low Temp. Phys. 39, 456 (2013)].

13. V.I. Iveronova and T.P. Revkevich, Theory of X-ray Scattering, Moscow University Publishers, Moscow (1978) [in Russian].

14. S.I. Kovalenko and N.N. Bagrov, Solid State Phys. 11, 2724 (1969).

15. S.S. Gorelik, L.N. Rastorguev, and Yu.A. Skakov, X-ray and Elecrtonoptical Analysis, Metallurgia, Moscow (1970) [in Russian].

16. A. Guinier, Theorie et Technique de la RadiocristalloGraphie, Dunod, Paris (1956).
17. Yu.F. Komnik, Fiz. Tver. Tela 6, 873 (1964).

18. D.W. Pashley, M.J. Stowell, and T.J. Law, Phys. Status Solidi 10, 153 (1965).

19. B.W. van de Waal, G. Torchet, and M.-F. Feraudy, Chem. Phys. Lett. 331, 57 (2000).

20. A.G. Danilchenko, S.I. Kovalenko, and V.N. Samovarov, Fiz. Nizk. Temp. 34, 1220 (2008) [Low Temp. Phys. 34, 996 (2008)].

21. V.V. Danchuk, A.A. Solodovnik, and M.A. Strzhemechny, Fiz. Nizk. Temp. 36, 321 (2010) [Low Temp. Phys. 36, 254 (2010)].

22. A.I. Gusev, Nanomaterials, Nanostructures, Nanotechnologies, FizMatLit, Moscow (2005) [in Russian].

23. S.V. Tsybula and S.V. Cherepanova, Introduction to the Structure Analysis of Nanocrystals, Novosibirsk University Publishers (2008) [in Russian].

24. A.G. Danilchenko, S.I. Kovalenko, and V.N. Samovarov, Fiz. Nizk. Temp. 30, 226 (2004) [Low Temp. Phys. 30, 166 (2004)]. 